\title{
Simulation of crack propagation in rocks by XFEM
}

\author{
G Sivakumar and VB Maji \\ Indian Institute of Technology Madras \\ Chennai, India \\ sivakumar.g516@gmail.com
}

\begin{abstract}
Understanding the failure mode and crack propagation in rock can provide useful information for safe and economic design of various structures in rock. Many researchers have developed theoretical criteria for rock failure with crack growth based on experimental observations. Numerically using cohesive zone model for brittle material with an assumption of some plasticity is found to be a good approach to predict the crack growth in rocks. The cohesive zone model is popularly used for fracture simulation in brittle materials, uses traction-separation law. The tractionseparation law represents the material damage zone in front of the crack tip where the material elements are pulled apart. The extended finite element method (XFEM) enhanced the capability of the classical finite element method capturing the crack propagation problems. The important feature of XFEM is that, it can extend the crack without any remeshing which makes it suitable for fracture process analysis. The present paper combines XFEM approach with cohesive zone model (CZM) to analyze the crack growth for rock using ABAQUS. The results of the analysis are compared with the experiments carried out in the laboratory and with available literatures on crack growth in rocks. The present paper demonstrates different crack types (tensile/shear) that gets initiated from the pre-existing flaw with respect to the applied loading. The numerical model using ABAQUS shows a good agreement with the theoretical and experimental results while predicting the crack propagation.
\end{abstract}

Keywords—cohesive zone model (CZM);XFEM;experimental studies;pre-existing flaw

\section{INTRODUCTION}

Rock and rock masses usually have discontinuity in the forms of cracks and fissures. To know the mechanical properties of rock in terms of strength and deformational behavior, rocks are mostly considered as homogeneous, isotropic and elastic materials. But assuming the rock in such a way, can't always give the correct strength and deformation characteristics as a material. This can be seen both in experimental study as well as numerical analysis, where the results of both usually be showing some overestimation. With the recent development in the numerical analysis, the study can be extended from intact rock properties to jointed rock material by considering the presence of the flaws/cracks. Many researchers attempted to study the rock having cracks and developed model based on the fracture mechanics concept [1-7]. The theory of fracture mechanics is useful to study the presence of the cracks and their effects on material property values. It can deal with the influence of micro cracks present in the material that will be leading to macroscopic failure. The present paper adopts the fracture mechanics theory to define the crack growth in rock and corresponding implementation and validation in the numerical model.

\section{STUDIES ON CRACK INITIATION AND PROPAGATION}

To understand the initiation and propagation of cracks in rock many researchers [1-5] conducted study based on laboratory experiments and numerical analysis. Most of them studied the brittle behavior in rock and found some common crack behaviour when subjected to loading. At first, primary wing cracks of tensile nature are seen and subsequently secondary cracks that appears after wing cracks of shear or tensile nature (Fig.1).

Lajtai [6] conducted uniaxial compression tests on plaster of Paris specimens containing single flaw of different dimensions and orientations with respect to the loading conditions. The results observed from the tests are shown in Fig. 2. The different crack patterns observed are (a) tensile crack which first appears after loading in a curvilinear path parallel to loading direction, (b) normal shear crack which initiated at the tip of the crack in a compressive stress area normal to loading direction, (c) additional shear zone formed around the crack tip when loading further applied and (d) inclined shear crack in the shear zone resulting in the loss of cohesive strength of material. Later [7] performed uniaxial compression tests on different materials such as glass, acrylic, plastic polymer and rocks having a single flaw [7] observed primary cracks get initiated at point of highest tensile stress and the secondary cracks originate from point of highest compressive stress concentration in rock. The crack growth pattern due to initiation and propagation of a single flaw occurred in a limestone under uniaxial loading condition found to have the primary (in tension nature) and secondary crack (in shear nature). The secondary cracks appear later after the primary crack in direction along the flaw tip and described as shear cracks for specimen failure.

With new experimental techniques involving the use of a high-speed camera [2] captured clear images of different crack propagations. Uniaxial compression tests are conducted on prismatic gypsum and Carrara marble specimens containing single flaws with varying inclination angles.

Seven different types of cracks observed with different mechanism initiated at the pre-existing flaw in the direction of the applied load as shown in Fig. 3. A study by [3] presents crack initiation, propagation and coalescence at or 


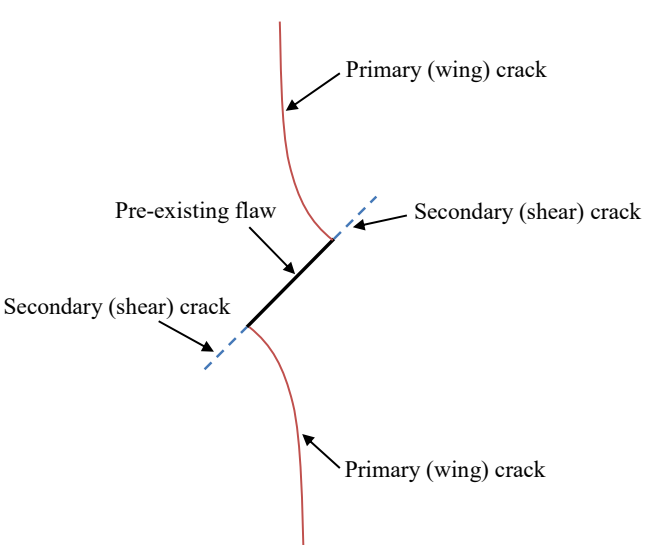

Fig.1. Primary and secondary cracks initiated from a pre-existing flaw

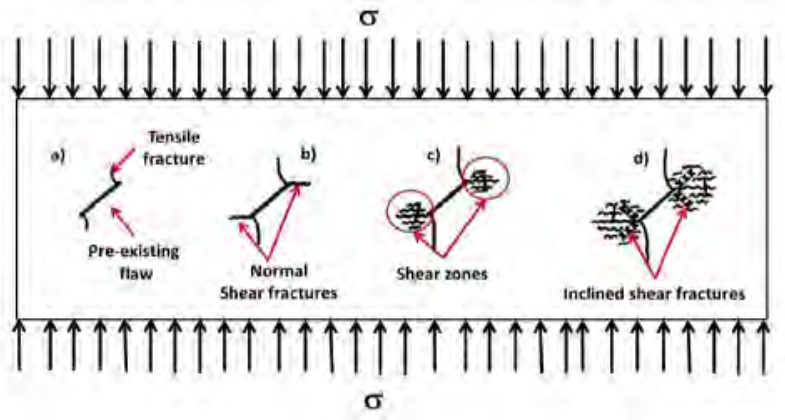

Fig.2. Fracture pattern observed from single flaw

near pre-existing open cracks or flaws in a specimen under uniaxial compression. Gypsum was used as a model material along with PMMA (Poly Methyl Methacrylate) and Hwangdeung granite. These experimental results are used for validating numerical simulations using Particle Flow Code in two dimensions (PFC2D).

Both experimental and numerical results are expected to improve the understanding of the characteristics of cracking and crack coalescence and can be used to analyze the stability of rock and rock structures, such as underground openings/slopes and tunneling constructions, where preexisting cracks or fractures play a crucial role in the overall integrity of the structures. Crack initiation and propagation in rocks has tremendous application in Hydro fracking. strengths of the materials. Griffith [9] originally postulated the theory on fracture initiation, which was based on energy considerations and the corresponding equations contained a surface traction term. The current interpretation of Griffith's theory is that, the fracture initiates when tensile stress induced at or near the tip of an inherent crack exceeds the molecular cohesive strength of the material [11].

With developments in the theories of fracture mechanism, there is a possibility of analyzing crack growth to describe their fracture process. The application of Griffith's criterion which is based on a genetic concept, namely the existence of small cracks or flaws to brittle rock has a theoretical foundation. $[8,9 \& 10]$ recognized that most brittle materials, such as glass, fail at applied stresses much lower than the theoretical.

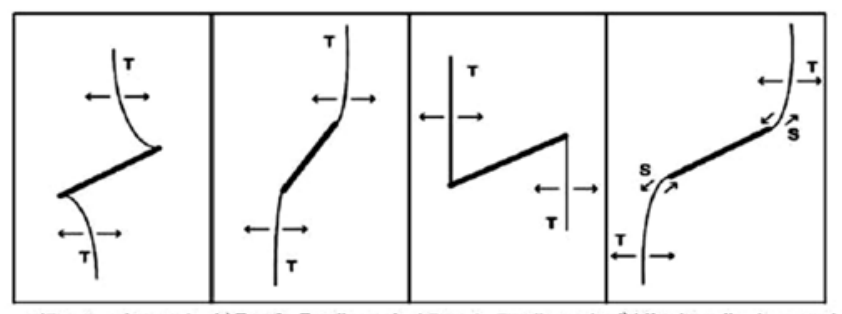

a)Type1 - wing crack b) Type2 - Tensile crack c) Type 3 - Tensile crack d) Mixed tensile-shear crack

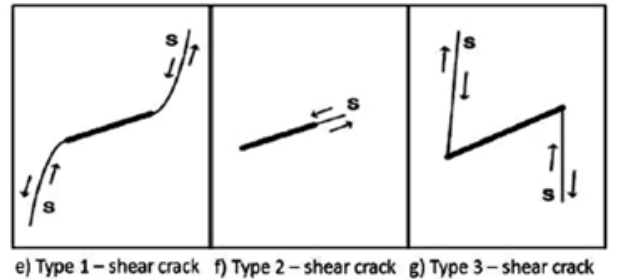

Fig.3. Crack types found in a single flaw under uniaxial compression

\section{Previous StUdies ON CRACK GROWTH MOdEL}

It is difficult to determine the molecular cohesive strength by direct measurement; instead the fracture criterion is expressed in terms of the uniaxial tensile strength of the material [12]. Later it was observed by [13] that, the Griffith hypothesis furnished a satisfactory criterion for fracture initiation, but the strength of the material cannot be predicted from fracture initiation criterion. Stated that [14], the theories like Mohr-Coulomb and Griffith were found to give poor agreement with the experimental results. Griffith theory has limitation that it can only be used to predict fracture initiation and doesn't yield any information on the rate or direction of fracture propagation. [17] noted that serious errors are generated, if fracture propagation considerations are not included in a fracture criterion.

For a better understanding of material failure behaviour in both macroscopic and microscopic sense, analysis at the crack tip is necessary. But the crack tip is surrounded by the fracture process zone (FPZ) where plastic deformation occurs. FPZ is the zone in which there is small scale yielding, micro-cracking and void initiation takes place. To represent the plastic deformation at the crack tip, [16] developed a microscopic plasticity model for ductile materials [17] independently developed a similar model for brittle materials considering molecular cohesion. The cohesive zone model which is a direct extension of the $[16,17]$ model considers the plasticity in linear elastic fracture mechanics. This consideration was implemented into finite element computations firstly by [18] for crack growth analysis of brittle materials. In the computational analysis, the material damage around the crack tip was simplified into a finite strip where the stress-strain curve for the zone is replaced by a traction separation law which will able to describe the behaviour of the damaging material ahead of the crack tip.

Many published studies on crack analysis used the cohesive zone model in [19]. But the cohesive zone model will have pre-assumption of the crack path in the regular FE models, where crack only propagates along the boundaries of elements. The applications of cohesive zone model are 
mainly limited to Mode-I loading cases, whereas in the actual engineering problems the materials are subjected to mixed-mode loading which usually advances along an unknown curved path [4]. Using extended finite element methods (XFEM), one can simulate crack nucleation at an arbitrary material point and crack propagation in an arbitrary direction without introducing extra nodes and elements. Moreover, it allows multi-cracks nucleation, growth and coalescence without remeshing.

\section{NUMERICAL MODELLING}

\section{A. Cohesive Zone Model}

The cohesive crack model assumes the existence of a fracture process zone (FPZ) in front of the crack tip (Fig. 4), in which the energy dissipation occurs during fracture. In the FPZ, there exist tractions in the normal directions and two tangential or shear directions across the crack surfaces, resulting from mechanisms such as material bonding, aggregate interlocking and surface friction.

In CZM, the material follows the traction-separation law used for defining the shear traction and crack sliding displacement relations across the crack tip. Per $\mathrm{Xu}$ and Needleman [20], before the first principal stress reaches the tensile strength, the material behaves linearly elastic. As soon the tensile strength is exceeded, the material begins to fail and the crack will get initiated. Crack initiation refers to the beginning of degradation of the cohesive response at an enriched element. The process of degradation begins when the stresses or the strains satisfy specified crack initiation criteria. The degradation of the material is controlled by damage evolution law which describes the rate at which the cohesive stiffness will be degraded once the corresponding initiation criterion is reached. To describe the evolution of damage across the interface of the material, fracture energy should be defined in the model.

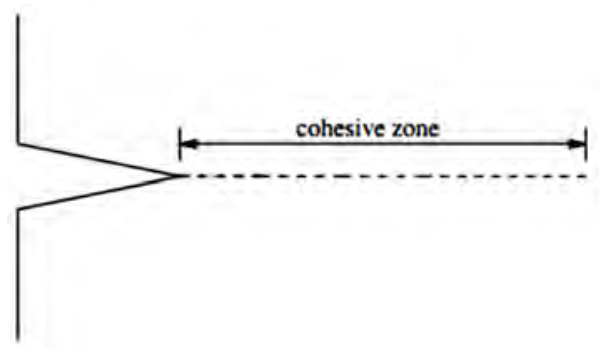

Fig.4. Schematic Representation of Cohesive Zone Model

When crack grows, the cohesive zone elements assigned in the mesh opens to simulate crack initiation. Since the crack path only follows the cohesive zone elements, crack propagation strongly depends on the mesh of the cohesive zone elements. This leads to the inclusion of Extended Finite Element Method (XFEM) approach, where the crack geometry is overlapped over the crack domain and their propagation happens without depending on the mesh.

\section{B. Basic concept of XFEM}

The extended finite element method (XFEM) is a numerical technique which extends the classical finite element method approach focusing on the crack propagation problems. It was first introduced by [21]. It is an extension of the conventional finite element method based on the concept of partition of unity, which allows local enrichment functions to be easily incorporated into a finite element approximation. The presence of discontinuities is ensured by the special enriched functions in conjunction with additional degrees of freedom. Simulation of propagating cracks with XFEM does not require initial crack and crack path definitions to conform to the structural mesh. The crack path is solution dependent i.e.; it is obtained as part of the solution.

For fracture analysis, the enrichment functions typically consist of the near-tip asymptotic functions that capture the singularity around the crack tip and a discontinuous function that represents the jump in displacement across the crack surfaces (equation 1). The approximation for a displacement vector function with the partition of unity enrichment is,

$$
u=\sum_{I=1}^{N} N_{I}(x)\left[u_{I}+H(x) a_{I}+\sum_{\alpha=1}^{4} F_{\alpha}(x) b_{I}^{\alpha}\right]
$$

where, NI (x) are the usual nodal shape functions; $\mathrm{uI}$ is the usual nodal displacement vector associated with the continuous part of the finite element solution; the second term is the product of the nodal enriched degree of freedom vector, a I and the associated discontinuous jump function $\mathrm{H}(\mathrm{x})$ across the crack surfaces; and the third term is the product of the nodal enriched degree of freedom vector, b I $\alpha$, and the associated elastic asymptotic crack-tip functions, $\mathrm{F} \alpha(\mathrm{x})$. In the present study, the near-tip asymptotic singularity is not required and only the displacement jump across a cracked element is considered.

\section{EXPERIMENTAL STUDIES}

For the experimental study, gypsum material is adopted to observe the crack growth process of the material. Based on the literature [1-3], gypsum is found to be an ideal model rock with which a wide range of brittle rocks can be represented and eases of preparing a significant number of specimens in a reasonable period. The experimental study is carried to confirm the effectiveness numerical model while capturing crack initiation and propagation in a specimen with pre-existing flaw.

The gypsum sample is prepared [3] having dimensions of $76 \mathrm{~mm}$ (width) $\times 152 \mathrm{~mm}$ (height) with a thickness varying from $25 \mathrm{~mm}$ to $30 \mathrm{~mm}$ and flaw size of $12.5 \mathrm{~mm}$ (length). To prepare this sample of this size a mold set up is fabricated and flaw is created with metal shims while casting. Metal shim is rotated as per the desired orientation of flaw from $0 \mathrm{o}$ to $90 \mathrm{o}$. The loading is performed in y-direction by uniaxial testing machine at a very slow rate of $0.031 \mathrm{~mm} / \mathrm{min}$ (Fig. 6). During the period of loading, the loading initiation of the crack and their crack propagation is captured until it reaches its maximum peak value. The observed crack growth pattern used to numerically implement crack growth. Uniaxial compression tests and Brazilian tensile tests were carried out to obtain mechanical properties of each material. Young's modulus, UCS (Uniaxial Compressive Strength), Poisson's ratio, and Brazilian tensile strength were obtained by averaging 3-5 laboratory test results, as shown in Table I. 
The specimen was loaded until it failed or cracks had coalesced under uniaxial compression. For each loading the crack initiation and the peak stress is recorded. The vertical displacements and applied load were recorded continuously during the period of loading. (obtained from uniaxial compression testing for defining

\section{Numerical ANALYSIS OF THE MODEL}

Present numerical analysis adopts XFEM approach combined with CZM to represent the crack growth process in rock by using the finite element tool ABAQUS. To describe CZM in ABAQUS, the elastic parameters Young's modulus and Poisson's ratio. initial elastic behavior of the un-cracked domain) is used for the study.

\section{TABLE I. MATERIAL PARAMETERS USED IN NUMERICAL MODEL}

\begin{tabular}{|l|l|}
\hline Material parameters & Values \\
\hline Young's modulus $(\mathrm{GPa})$ & 3.65 \\
\hline Uniaxial compressive strength $(\mathrm{MPa})$ & 35 \\
\hline Poisson's ratio & 0.34 \\
\hline Tensile strength $(\mathrm{MPa})$ & 0.82 \\
\hline Fracture energy $\left(\mathrm{J} / \mathrm{m}^{2}\right)$ & 24 \\
\hline
\end{tabular}

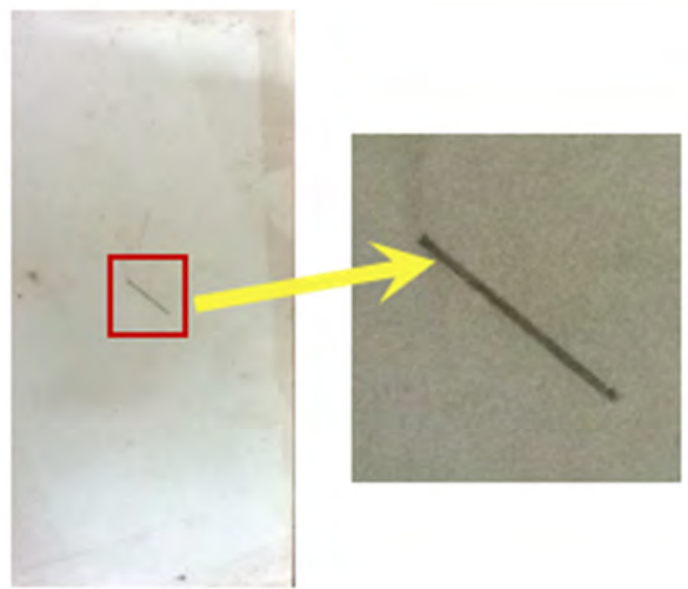

Fig.5. Artificial rock (gypsum plaster) specimen with inserted flaw

Until the crack gets initiated, the material adopts the elastic properties. Once the material strength reaches its material limit, it will behave based on traction-separation law.

Usually the material limit is considered when the maximum principal stress of the material reaches the tensile strength of the material. Therefore, the maximum tensile strength of the material is also needed to be determined from laboratory tests. The maximum principal stress criterion is used to define the crack initiation criteria [22]. To define the degradation of material, fracture energy is used in the numerical model to know the amount of energy been dissipated after the initiation at the crack tip and it is determined by ISRM suggested method [23].

The model is meshed in such a way that crack location must be contained within the crack domain. The mesh size

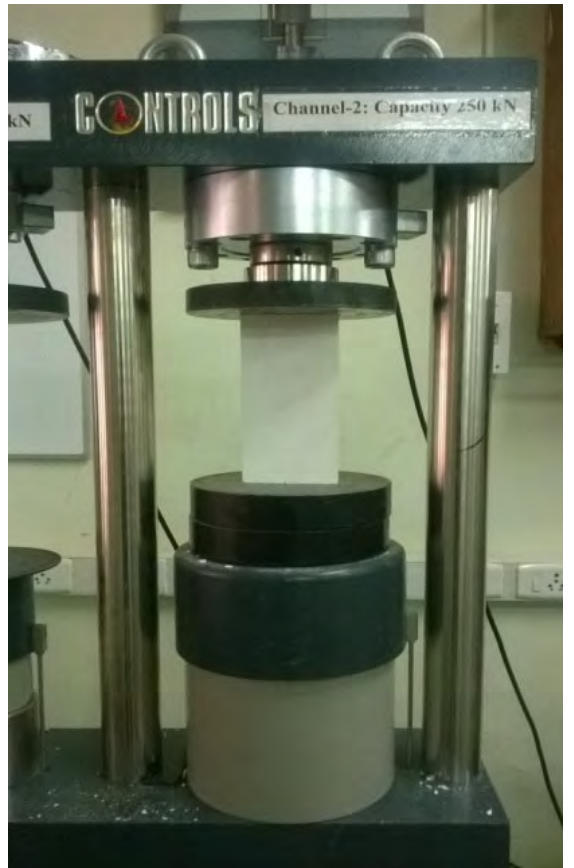

Fig.6. Experimental tests of gypsum sample conducted in uniaxial testing machine
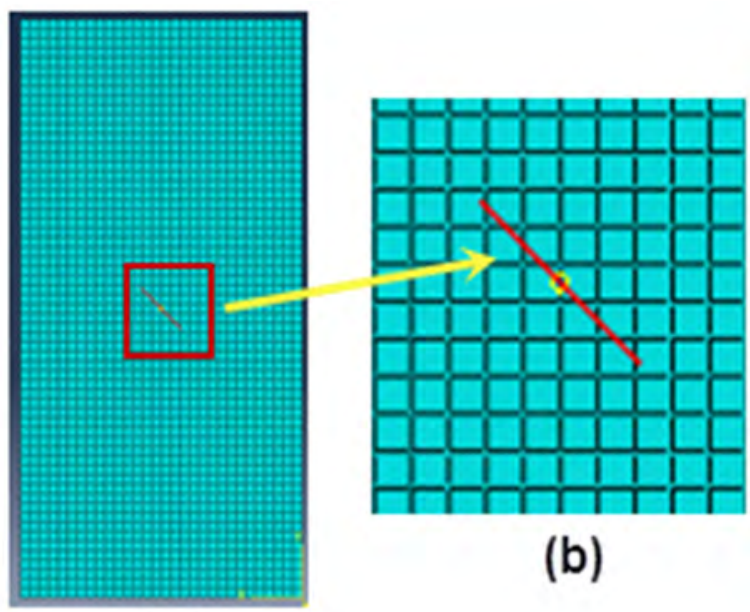

(b)

(a)

Fig.7. Numerical model in ABAQUS representing pre-existing flaw at $45^{\circ}$ angle with respect to the loading condition

for the model is made finer so that the propagation of the crack can be captured in a better manner (Fig. 7a). To select an element type for meshing a 4-node bilinear plane stress quadrilateral (CPS4R) is used. An interaction property is incorporated in model for the pre-existing flaw.

The boundary condition for the numerical model is chosen in accordance with the laboratory tests. The mesh size for the model is made finer so that the propagation of the crack can be captured in a better manner. The analysis is carried out in dynamic implicit quasi-static procedure and the loading is applied in a displacement controlled manner with a same loading rate of $0.031 \mathrm{~mm} / \mathrm{min}$ as applied in the experimental studies. 


\section{RESULTS AND OBSERVATIONS}

Some of the salient results and observations are discussed here. The developed numerical model found to can simulate the laboratory condition and the corresponding crack growth patterns. At the initial stage of the crack development, primary wing cracks appear to be of tensile nature (Fig.8). The cracks propagate in a stable manner following a curvilinear path parallel to the direction of the load applied. A shear zone around the crack tip is formed after the appearance of wing crack as the applied load increases. Later the shear zone expands around the crack tip resulting in the loss of cohesive strength in the material. The crack opening pattern in both experiments and numerical is found to be comparable. The wing crack propagation in analysis is as observed in the experimental studies. With refined mesh, proper crack propagation curvature towards the boundaries could be captured.

Fig.9 shows the comparison between the crack growth that has been observed in high speed camera after [3], present laboratory experiments and numerical analysis using XFEM analysis for some selected flaw orientation.

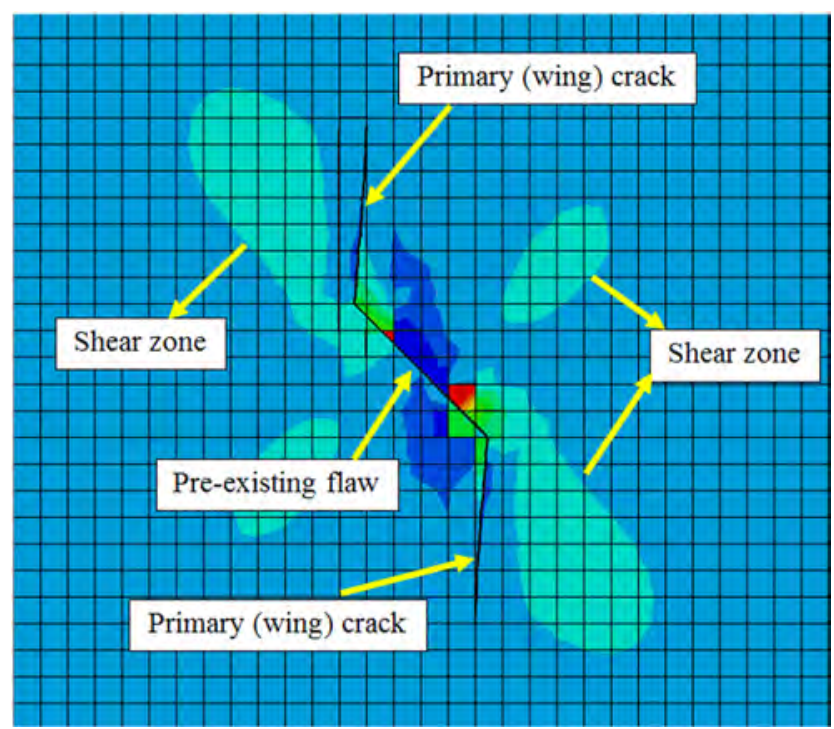

Fig.8. Crack observed in ABAQUS during process of loading

The crack initiation stress and their corresponding peak stress are noted down. It is found that the primary crack will start initiating sooner at the orientation angle of $45^{\circ}$ when compared to all other angles. But the peak stress found to be increasing with increase in the orientation of flaw angle. The primary cracks initiates with a flaw angle that varies from $15^{\circ}$ to $75^{\circ}$ and as the loading is increased, the wing cracks are observed to initiate with delay in the specimens with increase in flaw angle.

However, for the flaw angle greater than $75^{\circ}$, wing cracks are not observed as these cracks are difficult to initiate and propagate in this case. This could be due to the flaw's tendency to align to the parallel to the compressive loading direction. The stress concentration increases after the appearance of primary crack forming a shear zone around the crack tip. This formation of the shearzone

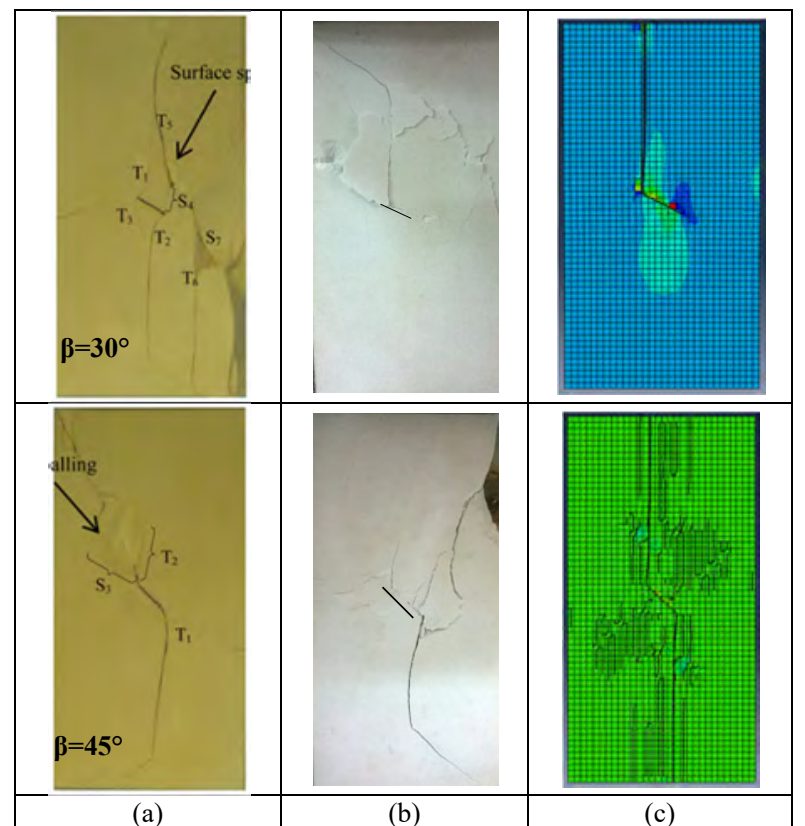

Fig.9. Comparison between the crack growths that has been observed in (a) high speed camera, (b) Present experiment (c) XFEM analysis

indicates the surface spalling that is been observed in the gypsum plaster in the laboratory experiments.

\section{SUMMARY AND CONCLUSIONS}

A numerical analysis has been carried out to capture the crack initialization and propagation of brittle rock with preexisting flaw subjected to uniaxial loading. Using FE software ABAQUS, the crack growth is realistically simulated with XFEM considering cohesive zone model (CZM). Laboratory experiments are conducted or artificial specimens with preexisting flaw and the results are used to validate the numerical analysis. The crack growth could be successfully captured in the analysis and the influence of peak stress on different flaw orientation respect to the applied loading is investigated. The numerical model shows a good agreement while predicting the shape and propagation of the crack. Failure criteria are crucial in this aspect and the selection of the appropriate damage criteria should be based on the available inputs derived from laboratory experiments. Further refinement in numerical simulation for better accuracy is under investigation.

\section{REFERENCES}

[1] A. Bobet and H.H. Einstein, "Numerical modeling of fracture coalescence in a model rock material,” Int. J. Fract., Vol. 92, pp. 221252, 1998.

[2] L.N.Y.Wong and H.H.Einstein, "Systematic evaluation of cracking behavior in specimens containing single flaws under uniaxial compression,” Int. J. Rock Mech. Min., Vol. 46, no. 2, pp. 239-249, 2009.

[3] H. Lee and S. Jeon, "An experimental and numerical study of fracture coalescence in pre-cracked specimens under uniaxial compression," Int. J. Solids Struct., Vol. 48, no. 6, pp. 979-999, 2011.

[4] Y. Xu and H. Yuan, "Applications of normal stress dominated cohesive zone models for mixed-mode crack simulation based on extended finite element methods," Eng. Fract. Mech., Vol. 78, pp. 544-558, 2011. 
[5] B. Gonçlaves da Silva and H.H. Einstein, "Modeling of crack initiation, propagation and coalescence in rocks," Int. J. Fract., Vol. 182, pp. 167-186, 2013.

[6] E. Lajtai, "A theoretical and experimental evaluation of the Griffith theory of brittle fracture," Tectonophysics, Vol. 11, no.2, pp. 129156, 1971.

[7] A.R. Ingraffea and F.E. Heuze, "Finite element models for rock fracture mechanics," Int. J. Numer. Anal. Met. Vol. 4, pp. 25-43, 1980.

[8] A.A. Griffith, "The phenomenon of rupture and flow in solids," Phil, Trans. R. Soc. Lond. A. 221, pp. 163-198, 1920.

[9] A.A. Griffith, "The theory of rupture," Proc. First Int. Cong. Appl. Mech., Delft, pp. 55-63, 1924.

[10] C. E. Inglis, "Stresses in a plate due to the presence of cracks and sharp corners," Proc. Inst. Naval Architects, Vol. 60, pp. 219-241, 1913.

[11] E. Orowan, "Fracture and strength of solids," Rep. Prog. Phys., Vol. 12, pp. $185-232,1949$

[12] E. Hoek, "Fracture of anisotropic rock," J. S. Afr. Inst. Min. Metall., 1964; Vol.64, no. 10, pp. 510-18.

[13] E. Hoek and Z.T. Bieniawski, "Brittle fracture propagation in rock under compression,” J. Fract. Mech., Vol. 1, no. 3, pp. 139-155, 1965.

[14] Z.T. Bieniawski, "Mechanism of brittle fracture of rock, Part I, II and III,” Int. J. Rock Mech. Min., Vol. 4, no. 4, pp. 395-430, 1967.

[15] G.I. Barenblatt, "Brittle Fracture," Royal Institute of Technology Publications, StockhoLm, no. 149, 1966.

[16] D.S. Dugdale, "Yielding of steel sheets containing slits," J. Mech. Phys. Solids, Vol. 8, pp. 100-104, 1960.

[17] G.I. Barenblatt, "The mathematical theory of equilibrium of cracks in brittle fracture," Adv. Appl. Mech., Vol. 7, pp. 55-129, 1962.

[18] A. Hillerborg, M. Modeer and P.E. Petersson, "Analysis of crack formation and crack growth in concrete by means of fracture mechanics and finite elements," Cement and Concrete Res., Vol. 6, pp. 773-782, 1976.

[19] ABAQUS Theory Manual, Version 6.12. ABAQUS, Inc. Providence, R.I., 2012.

[20] X.P. Xu and A. Needleman, "Numerical simulations of fast crack growth in brittle solids," J. Mech. Phys. Solids, Vol. 42, pp. 13971434, 1994.

[21] T. Belytschkoand T. Black,"Elastic crack growth in finite elements with minimal remeshing,"'Int. J. Numer. Meth. Eng., Vol. 45, pp. 601620, 1999.

[22] G. Sivakumar and V.B. Maji, "Finite element simulation of crack initiation and propagation in rocks," Eurock-2014: ISRM European Regional Symposium, 26-28 May 2014, Vigo, Spain, 2014.

[23] ISRM, "International Society for Rock Mechnaics (ISRM)suggested method for determining mode I fracture toughness using cracked chevron notched Brazilian disk (CCNBD) specimens," Int. J. Rock. Mech. Min. Sci., Vol. 32, no.1, pp.57-64, 1995. 